\title{
Escala de Usabilidade do Sistema Baseada na Lógica Paraconsistente Anotada
}

\section{Evidencial $\mathbf{E} \tau$}

\author{
System Usability Scale Based on Paraconsistent Annotated Evidential Logic E $\tau$ \\ Escala de Usabilidad del Sistema Basada en la Logica Paraconsistente Anotada Evidencial E $\tau$
}

Recebido: 27/06/2021 | Revisado: 07/07/2021 | Aceito: 07/07/2021 | Publicado: 18/07/2021

Luiz Roberto Forçan
ORCID: https://orcid.org/0000-0001-7376-830X
Universidade Paulista, Brasil
E-mail: luforcan@gmail.com
Jair Minoro Abe
ORCID: https://orcid.org/0000-0003-2088-9065
Universidade Paulista, Brasil
E-mail: jairabr@uol.com.br
Samira Sestari do Nascimento
ORCID: https://orcid.org/0000-0001-7429-6033
Universidade Paulista, Brasil
E-mail: sestarisamira@gmail.com
Cristina Corrêa de Oliveira
ORCID: https://orcid.org/0000-0002-8629-6679
Instituto Federal de São Paulo, Brasil
E-mail: crisolive@ifsp.edu.br

\section{Resumo}

A opinião da experiência do usuário utilizando um software afeta diretamente a qualidade dele. A Escala de Usabilidade do Sistema mensura a experiência do usuário final com o sistema, que apresenta questões que se contradizem. O objetivo deste artigo é demonstrar um aprofundamento das contradições, aplicando a Lógica Paraconsistente Anotada Evidencial $\mathrm{E} \tau$, com a realização de uma pesquisa com onze profissionais que avaliaram um sistema. Foi realizada uma comparação dos resultados da escala SUS com o algoritmo Para-analisador, utilizando o $\operatorname{par}(\mu ; \lambda)$, atribuindo as questões pares para $\mu$ e as questões ímpares para $\lambda$. O primeiro resultado apresentou uma avaliação positiva da usabilidade, com a Escala de Usabilidade do Sistema (76,59). O segundo resultado, realizado com o Para-analisador, apresentou a média global $(0,75 ; 0,25)$, que aponta uma similaridade com o primeiro, destacando dois itens contraditórios. A avaliação aprimorou a análise da usabilidade, pois houve a concordância dos respondentes em nove características da usabilidade. Porém apresentou uma divergência em um item, visto que cinco respondentes avaliaram duas questões contraditórias com valores altos, apresentando uma contradição na avaliação. Este resultado pode evidenciar uma falha de percepção na semântica do instrumento, que poderia indicar uma necessidade de treinamento dos participantes antes do início do processo. A principal contribuição desta pesquisa foi avaliar a usabilidade de um software em conjunto com o Paraanalisador, possibilitando uma análise mais detalhada, pois ele trabalha com o par $(\mu ; \lambda)$, sendo possível avaliar os resultados em duas dimensões.

Palavras-chave: Escala de usabilidade do sistema; Lógica paraconsistente Anotada Evidencial E $\tau$; Contradição; Análise bidimensional.

\begin{abstract}
The evaluation of the user experience using a software directly affects its quality. The System Usability Scale measures the end user's experience with the system, which presents contradictory issues. The objective of this article is to demonstrate a deepening of those contradictions, using the Evidential Annotated Paraconsistent Logic E $\tau$, with the application of a survey for eleven professionals who evaluated a system. A comparison of the results of the above scale was performed with the Para-analyzer algorithm, using the pair $(\mu, \lambda)$, assigning the even questions to $\mu$ and the odd questions to $\lambda$. The first result showed a positive assessment of usability, with the System Usability Scale (76.59). The second result, performed with the Para-analyzer, presented the global average $(0.75,0.25)$, which indicates a similarity with the first, highlighting two contradictory items. The evaluation improved the usability analysis, as respondents agreed on nine usability characteristics. However, there was a divergence in one item, as five respondents rated two questions with high values, presenting a contradiction in the assessment. This result may show a perception failure in the instrument's semantics, which could indicate a need for training of participants before the beginning of the process. The main contribution of this research was to evaluate the usability of a software in conjunction with the Para-analyzer, enabling a more detailed analysis, as it works with the pair $(\mu, \lambda)$, being possible to evaluate the results in two dimensions.
\end{abstract}

Keywords: System usability scale; Paraconsistent Logic Annotated E $\tau$; Contradiction; Two-dimension analysis. 


\section{Resumen}

La evaluación de la experiencia del usuario al utilizar un software afecta dedirectamente a su calidad. La escala de usabilidad del sistema mide la experiencia del usuario final con el sistema, que presenta problemas contradictorios. El objetivo de este artículo es demostrar una profundización de esas contradicciones, utilizando la Logica Paraconsistente Anotada Evidencial E $\tau$, con la aplicación de una encuesta a once profesionales que evaluaron un sistema. Se realizó una comparación de los resultados de la escala anterior con el algoritmo Para-analizador, utilizando el par $(\mu, \lambda)$, asignando las preguntas pares a $\mu$ y las preguntas impares a $\lambda$. El primer resultado mostró una valoración positiva de la usabilidad, con la Escala de usabilidad del sistema (76,59). El segundo resultado, realizado con el Paraanalizador, presentó el promedio global $(0,75,0,25)$, lo que indica similitud con el primero, destacando dos ítems contradictorios. La evaluación mejoró el análisis de usabilidad, ya que los encuestados coincidieron en nueve características de usabilidad. Sin embargo, hubo una divergencia en un ítem, ya que cinco encuestados calificaron dos preguntas con valores altos, lo que presenta una contradicción en la evaluación. Este resultado puede mostrar una falla de percepción en la semántica del instrumento, lo que podría indicar la necesidad de capacitar a los participantes antes del inicio del proceso. El principal aporte de esta investigación fue evaluar la usabilidad de un software en conjunto con el Para-analizador, permitiendo un análisis más detallado, ya que trabaja con el par $(\mu, \lambda)$, siendo posible evaluar los resultados en dos dimensiones.

Palabras clave: Escala de usabilidad del sistema; Logica Paraconsistente Anotada Evidencial E $\tau$; Contradicción; Análisis bidimensional.

\section{Introdução}

A competitividade da indústria de software obriga os desenvolvedores a aprimorarem o processo de produção de software a fim de obter vantagens competitivas, pois a qualidade de um software está diretamente ligada ao modelo de processo de software. Avaliação da usabilidade mede o grau de satisfação dos usuários que utilizam um sistema no seu dia a dia, denominado Sistema de Gestão de Prestadores de Serviço (SGPS).

A norma ISO/IEC 25010 (ISO/IEC 25010, 2021), define características e subcaracterísticas, internos e externos da qualidade de um software e seu uso. A Usabilidade se divide em seis subcaracterísticas: Inteligibilidade, Apreensibilidade, Operacionalidade, Proteção contra Erros do usuário, Estética da Interface e Acessibilidade (Esaki et al., 2013).

Existem diversos instrumentos de avaliação da usabilidade (Jeff Sauro, 2016); a Escala de Usabilidade do Sistema (SUS) (Brooke, 1995) avalia a experiência do usuário com poucos itens, sendo aplicados em testes de usabilidade, capacidade de aprendizado, entre outros aspectos. As principais vantagens do SUS são a objetividade na coleta de dados primários resultante da interação dos usuários com o sistema; na replicabilidade do instrumento; e na facilidade de quantificação e análise dos resultados.

A Lógica Paraconsistente Anotada Evidencial E $\tau$ (Lógica E $\tau$ ), permite a análise das opiniões subjetivas dos usuários, considerando suas incertezas e contradições, que são inerentes aos valores humanos (Abe, 2015). Está lógica faz parte do grupo das lógicas não clássicas.

A pesquisa apresenta uma proposta de restruturação interna do SUS, utilizando o algoritmo Para-analisador, sendo que as variáveis de entrada foram estabelecidas pelo julgamento dos usuários do sistema. A Lógica E $\tau$ utiliza critérios lógicos que permite evidenciar a contradição, auxiliando no processo decisório.

O procedimento deste estudo foi um survey com onze profissionais da área administrativa, de uma empresa de assistência médica privada, utilizando o SUS, a fim de mensurar a usabilidade do Sistema de Gestão de Prestadores de Serviços, apresentando os resultados com o SUS, comparando-os com uma adaptação interna, tendo como base a Lógica E $\tau$, atribuindo as respostas ao par $(\mu, \lambda)$, a fim de ressaltar as contradições.

\section{Referencial Teórico}

Os seguintes tópicos estão fundamentados na teoria sobre Avaliação da Qualidade de Software, Sistema de Gestão de Prestadores de Serviço, Escala do Usabilidade de Sistema, assim como a aplicação da Lógica Paraconsistente Anotada Evidencial $\mathrm{E} \tau$. 


\subsection{Qualidade de Software}

Qualidade é um termo relativo, para uma pessoa um produto pode ter qualidade, para outra, a qualidade pode estar ausente (Denning, 2016). No desenvolvimento de software, a qualidade e a confiabilidade são dois tópicos importantes (ISO/IEC 25010, 2021), pois as métricas mensuram as características internas e externas de um software após uma avaliação subjetiva realizado por humanos reagindo a artefatos.

Devido ao fato do desenvolvimento de software ser centrado no ser humano, ignorar as práticas de qualidade e o processo de avaliação dos aspectos psicológicos e comportamentais na usabilidade podem acarretar em consequências sociais, organizacionais, econômicas e provocar o fracasso do produto de software (Ghanbari et al., 2018).

A qualidade do software precisa ser garantida pelo padrão de qualidade do software, avaliação da qualidade do software e mecanismo de gerenciamento do processo de testes de software (Shen et al., 2018).

A norma ISO/IEC 25040 apresenta uma descrição do processo de aferição da qualidade de um software, estabelecendo os requisitos para esta atividade (ISO/IEC 25040, 2021). As atividades de avaliação da qualidade de um software devem ser projetadas antecipadamente, considerando-se a disponibilidade de recursos, ferramentas de software e instrumentos de avaliação. O plano de avaliação também deve incluir o propósito da avaliação, os padrões, os métodos e ferramentas (Brooke, 1995).

\subsection{Sistema de Gestão de Prestadores de Serviço}

O Sistema de Gestão de Prestadores de Serviços (SGPS) foi desenvolvido utilizando a metodologia de gerenciamento ágil Scrum (Kniberg, 2015) com o objetivo de automatizar o workflow de contratação de prestadores de serviço, a fim de realizar o gerenciamento eletrônico de dados reduzindo os custos do processo e melhorando a eficiência operacional seguindo a abordagem de resolução de problemas (Laudon, 2014).

Três profissionais daquela área desempenharam o papel de Product Owners, participando do desenvolvimento, junto com a área de Tecnologia da Informação. Para Manchar \& Chouhan (2017), para o desenvolvimento do SGPS foi utilizado a ferramenta SalesForce ${ }^{\circledR}$, que tem como característica principal a facilidade da gestão de relacionamento com clientes Customer Relationship Management (Buyya et al., 2011).

\subsection{Escala de Usabilidade do Sistema}

A Escala de usabilidade do sistema (SUS) é utilizada na análise da usabilidade, pois tem se mostrado um instrumento flexível, não sendo afetado por mudanças de palavras e linguagem (Wahyuningrum et al., 2020).

O SUS é composto por 10 questões padronizadas (Brooke, 1995), onde, para cada uma das questões, o usuário elege uma resposta que está atribuída a um modo de distribuição escalar, com valores variando de um a cinco, nos extremos entre: "Concordo Totalmente" até "Discordo Totalmente", conforme pode ser visto na Tabela 1.

Confiabilidade e usabilidade são dois tópicos importantes em um software conforme a norma ISO/IEC 25010 (2021). A usabilidade é a ideia central da interação homem-computador, pois a experiência do usuário combina aspectos como eficiência e eficácia com critérios adicionais como qualidade, facilidade de uso e atratividade.

Equação 1 - Fórmula do SUS.

SUS $=\left[\sum_{n=1}^{5}\left(\mathrm{U}_{2 n-\mathbf{1}}-\mathbf{1}+\left(\mathbf{5}-\mathrm{U}_{2 n}\right)\right] \times \mathbf{2 . 5}\right.$

Fonte: Brooke (1995). 
O resultado final do SUS é a soma de todas as contribuições da pontuação para os dez itens multiplicados por 2.5, conforme mostrado em Equação 1, onde $U_{i}$ se refere à classificação do i-ésimo item, variando entre 0 e 100, onde valores mais altos refletem maior satisfação do usuário.

Resultados com pelo menos 90 pontos representa que a expectativa, na usabilidade, está acima do esperado pelo usuário; valores entre 80 e 90 pontos representam usabilidade excelente. Os resultados que atingem entre 70 e 80 pontos são os que apresentam boa usabilidade, mas que apresentam algumas melhorias a serem realizadas. Os que atingem resultado entre 60 e 70 pontos são considerados "ok" mas devem ser fortemente melhorados, e por fim, os abaixo de 60 pontos não apresentam grau de usabilidade aceitável (Brooke, 1995).

Tabela 1 - Escala de usabilidade do sistema.

\begin{tabular}{|c|c|}
\hline Questões & Critério \\
\hline $\begin{array}{l}\text { Q1 - Eu acho que gostaria de usar essa aplicação frequentemente. } \\
\text { Q2 - Eu achei essa aplicação desnecessariamente complexa. } \\
\text { Q3 - Eu achei a aplicação fácil para usar. } \\
\text { Q4 - Eu acho que precisaria do apoio de um suporte técnico para usar essa aplicação. } \\
\text { Q5 - Eu achei que as várias funções da aplicação estavam bem integradas. } \\
\text { Q6 - Eu achei que havia muita inconsistência na aplicação. } \\
\text { Q7 - Imagino que a maioria das pessoas possa aprender a utilizar este aplicativo muito rapidamente. } \\
\text { Q8 - Achei a aplicação muito complicada de se usar. } \\
\text { Q9 - Eu me senti muito confiante em utilizar esta aplicação. } \\
\text { Q10 - Eu precisei aprender várias coisas antes que eu pudesse começar a usar essa aplicação. }\end{array}$ & 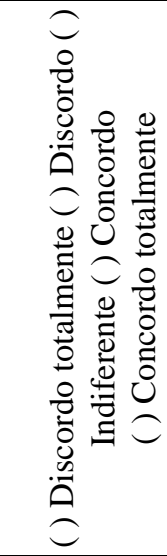 \\
\hline
\end{tabular}

Fonte: Brooke (1995).

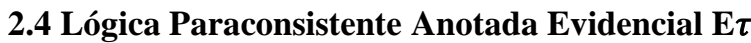

A tomada de decisão envolve a congruência das opiniões dos membros participantes, na seleção de opções alternativas (Abe et al., 2011). Em geral, a tomada de decisão é baseada num processo cognitivo que envolvem aspectos subjetivos e intuitivos. A intuição é um ato inconsistente e involuntário e a subjetividade não tem uma medida perfeita. A coleta de informações advém de diversas fontes, podendo ser muitas vezes contraditórias, abrindo lacunas para as incertezas e contradições que impedem, muitas vezes, a tomada de decisão (Akama, 2016).

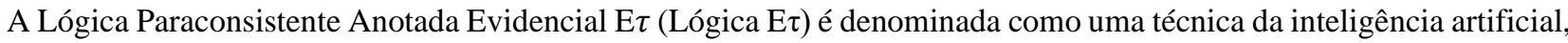
pois pertence as chamadas lógicas não clássicas, em razão de possuir premissas contraditórias como o princípio da contradição (Abe, 2015), que diferentemente da lógica clássica, uma sentença e a sua negação podem ser ambas verdadeiras.

A Lógica E $\tau$ permite tratar dados subjetivos do mundo real em dados precisos com saídas numéricas (Abe et al., 2015). Uma de suas vantagens é realizar a tradução da linguagem natural (termos linguísticos) utilizada na comunicação cotidiana em expressões matemáticas (Abe., 2015). Isso é obtido por meio de propriedades de anotações reticuladas, que cada preposição $p$ associa, no senso comum, um par $(\mu ; \lambda)$, representado pelas letras gregas mi $(\mu)$ e lambda $(\lambda)$, descrito da seguinte maneira: $p$ $(\mu ; \lambda)$ denominado constante de anotação, que pertence ao produto Real Cartesiano $[0,1]$ x $[0,1]$. Intuitivamente, $\mu$ representa o grau de evidência favorável expresso em $\mathrm{p}$, e $\lambda$ representa o grau de evidência desfavorável expresso por p. Os valores de $\mu$ e $\lambda$ estão limitados entre 0 e 1 (Abe, 2015).

$\mathrm{Na}$ Lógica $\mathrm{E} \tau$, os estados da decisão possuem quatro valores limites extremos: verdadeiro, falso, inconsistente e paracompleto (Da Silva Filho et al., 2008). 
- $\quad$ O estado verdadeiro (V) acontece quando $\mu=1,0$ e $\lambda=0,0$

- $\quad$ O estado falso (F) acontece quando $\mu=0,0$ e $\lambda=1,0$

- $\quad$ O estado inconsistente (T) acontece quando $\mu=1,0$ e $\lambda=1,0$

- $\quad$ O estado paracompleto $(\perp)$ acontece quando $\mu=0,0$ e $\lambda=0,0$

Além dos quatro estados extremos, possui também oito estados não extremos, conforme Tabela 2, que são denominados de acordo com a proximidade com os estados lógicos extremos conforme Figura 1, como possíveis resultados para um suporte fundamental no momento da tomada de decisão permitindo mais possibilidades de análise (Abe, 2010).

Figura 1 - Representação do

Reticulado $\tau$.

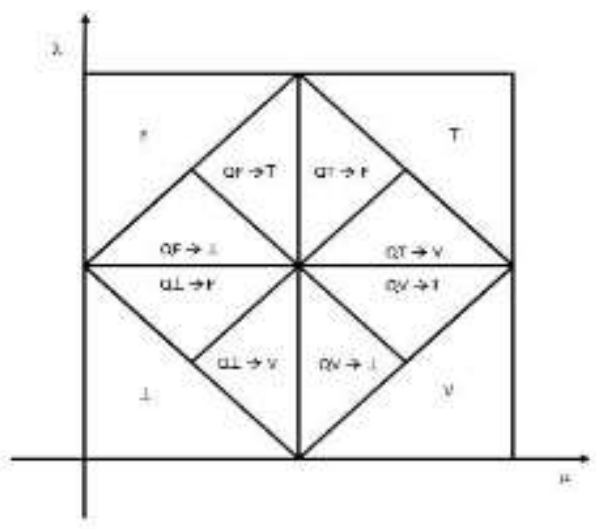

Tabela 2 - Simbolização dos Estados Lógicos.

\begin{tabular}{lc|lc}
\hline Estados extremos & \multicolumn{1}{c}{ Símbolo } & Estados não extremos & Símbolo \\
\hline Verdadeiro & $\mathrm{V}$ & Quase-Verdade tendendo a Inconsistente & $\mathrm{Q}_{\mathrm{V}} \rightarrow \mathrm{T}$ \\
Falso & $\mathrm{F}$ & Quase-Verdade tendendo a Paracompleto & $\mathrm{Q}_{\mathrm{V}} \rightarrow \perp$ \\
Inconsistente & $\mathrm{T}$ & Quase-Falso tendendo a Inconsistente & $\mathrm{Q}_{\mathrm{F}} \rightarrow \mathrm{T}$ \\
Paracompleto & $\perp$ & Quase-Falso tendendo a Paracompleto & $\mathrm{Q}_{\mathrm{F}} \rightarrow \perp$ \\
& & Quase-Inconsistente tendendo a Verdadeiro & $\mathrm{Q}_{\mathrm{T}} \rightarrow \mathrm{V}$ \\
& Quase-Inconsistente tendendo a Falso & $\mathrm{Q}_{\mathrm{T}} \rightarrow \mathrm{F}$ \\
& & Quase-Paracompleto tendendo a & $\mathrm{Q}_{\perp} \rightarrow \mathrm{V}$ \\
& & Verdadeiro & $\mathrm{Q}_{\perp} \rightarrow \mathrm{F}$ \\
\hline
\end{tabular}

Fonte: Abe (2015).

O algoritmo Para-analisador auxilia a tomada de decisão, pois ele admite a evidência favorável e desfavorável, advindas de um instrumento de avaliação que capta opiniões, auxiliando a tomada de decisão (Carvalho \& Abe, 2018). A Figura 1 apresenta o Quadrado Unitário do Plano Cartesiano (QUPC), sendo que o par $(\mu ; \lambda)$ representa a evidência favorável e desfavorável. Os graus de certeza e de contradição são calculados pelos operadores OR e AND, conforme pode ser visto na Figura 2, dado que o OR representa a maximização das opiniões dos especialistas, e o AND representa a minimização das opiniões dos especialistas. O cômputo final, denominada Análise Global, é a média dos graus resultantes, que pode apresentar doze estados, sendo que são quatro extremos e oito não extremos (Abe et al., 2015). 
Figura 2 - Lógica Et evidenciando a contradição.

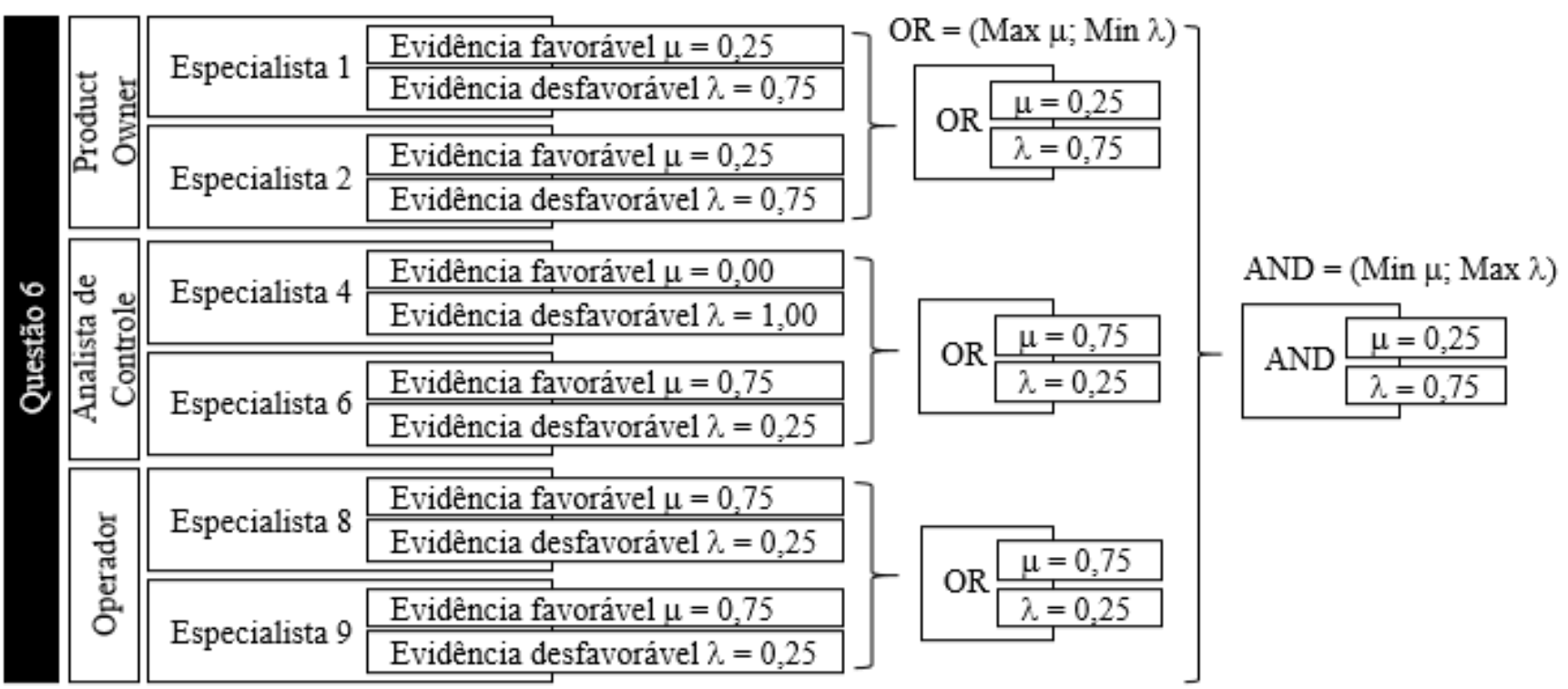

Fonte: Autores.

\section{Metodologia}

A presente pesquisa é de natureza aplicada, com objetivo exploratório e de abordagem quali-quantitativa (Pereira et al., 2018), pois se pretende aplicar Lógica E $\tau$ na captação de contradições dos respondentes na avaliação da usabilidade do SGPS. Esta seção apresenta os procedimentos aplicados neste estudo, com o propósito de ressaltar as contradições nos resultados. Foram estabelecidos, no enquadramento metodológico, o tipo de pesquisa; o objetivo da pesquisa; o procedimento da pesquisa com a definição dos usuários; a definição do instrumento e coleta da avaliação de usabilidade e, finalmente, a análise dos dados.

Esta pesquisa é de natureza aplicada, pois busca-se gerar conhecimento para a aplicação prática e dirigida à solução de problemas na usabilidade de software, pois está é um fator preponderante na aceitação de um software, pois sem ela o usuário não tem como desenvolver suas tarefas adequadamente (Harrison et al., 2013).

O objetivo deste estudo é exploratório, pois almeja-se ressaltar a contradição, com a Lógica Et, na avaliação de usabilidade aplicando o SUS; sendo que as perguntas são fechadas, utilizando uma escala Likert (Stevens, 1936), com valores normalizados variando de cinco a um, nos extremos com: "Concordo totalmente" a "Discordo totalmente", correspondendo aos critérios numéricos atribuídos a escala, conforme Tabela 3.

Tabela 3 - Normalização de $\mu$ e $\lambda$.

\begin{tabular}{lll}
\hline Resposta & Score SUS & Valor Lógica E $\tau$ \\
\hline Concordo totalmente & 5 & 1,00 \\
Concordo & 4 & 0,75 \\
Indiferente & 3 & 0,50 \\
Discordo & 2 & 0,25 \\
Discordo totalmente & 1 & 0,00 \\
\hline
\end{tabular}

Fonte: Autores.

O procedimento adotado por este artigo, foi um survey, pois busca-se informações com um grupo de interesse, a fim de capturar a percepção dos usuários, aplicando um instrumento de avaliação para a população alvo de onze profissionais da área de Gerência de Prestadores de Serviço, que emitiram suas opiniões sobre a usabilidade do software utilizado em sua rotina diária. 
$\mathrm{O}$ anonimato dos participantes, que receberam o instrumento de avaliação por meio eletrônico, foi mantido. Eles foram organizados em três grupos, adotando o cargo como critério de classificação sendo o Product Owner (PO), os Analistas de Controle (AC) e os Operadores (OP), conforme Tabela 4. Eles foram denominados $\mathrm{E}_{1}, \mathrm{E}_{2}, \ldots, \mathrm{E}_{11}$ na base de dados.

Tabela 4 - Classificação dos Grupos de usuários.

\begin{tabular}{lll}
\hline Grupo & Ocupação & Número de entrevistados \\
\hline A & Product Owner & 3 \\
B & Analista de Controle & 4 \\
C & Operador & 4 \\
\hline
\end{tabular}

Fonte: Autores.

A coleta de dados primários foi realizada com o SUS. A Tabela 5 apresenta uma pequena amostra da base de dados da avaliação da usabilidade, sendo que as respostas foram atribuídas da seguinte forma: ímpares para evidência favorável $(\mu)$ complementando o valor de $\lambda$ com $1-\mu$, e as pares foram atribuídas para a evidência desfavorável $(\lambda)$, complementando o valor de $\mu$ com $1-\lambda$. A evidência favorável e a evidência desfavorável são valores que pertencem ao intervalo [0,1], e resultam no grau de certeza (Abe, 2015).

Por exemplo, na questão Q1 do Especialista 1, foi respondido concordo, que após a normalização equivale ao valor 0,75 na Lógica Et, atribuído a $\mu$ e com $\lambda$ complementar 0,25 , formando o par $(0,75 ; 0,25)$. A partir da aplicação desta regra elaborouse a Tabela 5:

Tabela 5 - Base de dados formada $\mu$ e $\lambda$ atribuídos pelos usuários.

\begin{tabular}{|c|c|c|c|c|c|c|c|c|c|c|c|c|c|c|c|c|c|c|c|c|c|c|}
\hline \multirow{3}{*}{ Questão } & \multicolumn{6}{|c|}{ Product owner } & \multicolumn{8}{|c|}{ Analista de Controle } & \multicolumn{8}{|c|}{ Operador } \\
\hline & \multicolumn{2}{|c|}{ El } & \multicolumn{2}{|c|}{ E2 } & \multicolumn{2}{|c|}{ E3 } & \multicolumn{2}{|c|}{ E4 } & \multicolumn{2}{|c|}{ E5 } & \multicolumn{2}{|c|}{ E6 } & \multicolumn{2}{|c|}{ E7 } & \multicolumn{2}{|c|}{ E8 } & \multicolumn{2}{|c|}{ E9 } & \multicolumn{2}{|c|}{ E10 } & \multicolumn{2}{|c|}{ E11 } \\
\hline & $\mu$ & $\lambda$ & $\mu$ & $\lambda$ & $\mu$ & $\lambda$ & $\mu$ & $\lambda$ & $\mu$ & $\lambda$ & $\mu$ & $\lambda$ & $\mu$ & $\lambda$ & $\mu$ & $\lambda$ & $\mu$ & $\lambda$ & $\mu$ & $\lambda$ & $\mu$ & $\lambda$ \\
\hline Q1 & 0,75 & $0,25 \mid$ & 0,75 & 0,25 & 1,00 & 0,00 & 1,00 & 0,00 & 1,00 & 0,00 & 1,00 & 0,00 & 1,00 & 0,00 & 0,75 & $0,25 \mid$ & 1,00 & 0,00 & 0,75 & 0,25 & 0,75 & 0,25 \\
\hline
\end{tabular}

Fonte: Autores.

\section{Resultados e Discussão}

O resultado do SUS foi calculado aplicando a Equação 1, conforme pode ser conferido na coluna SUS Score da Tabela 6, sendo que a população alvo foi constituída por onze usuários que avaliaram o SGPS.

Tabela 6 - SUS Cálculo da pontuação total.

\begin{tabular}{llllllllllll}
\hline Especialista & Q1 & Q2 & Q3 & Q4 & Q5 & Q6 & Q7 & Q8 & Q9 & Q10 & SUS Score \\
\hline E1 & 4 & 2 & 4 & 2 & 4 & 4 & 2 & 2 & 4 & 4 & 60,0 \\
E2 & 4 & 2 & 4 & 2 & 4 & 4 & 4 & 1 & 4 & 2 & 72,5 \\
E3 & 5 & 2 & 4 & 1 & 4 & 4 & 4 & 1 & 4 & 2 & 77,5 \\
E4 & 5 & 2 & 4 & 4 & 4 & 5 & 4 & 2 & 4 & 2 & 65,0 \\
E5 & 5 & 1 & 5 & 1 & 5 & 5 & 5 & 1 & 2 & 1 & 82,5 \\
E6 & 5 & 2 & 5 & 2 & 4 & 2 & 4 & 2 & 5 & 2 & 82,5 \\
E7 & 5 & 1 & 5 & 1 & 4 & 2 & 5 & 1 & 4 & 2 & 90,0 \\
E8 & 4 & 1 & 5 & 2 & 3 & 2 & 4 & 2 & 4 & 1 & 80,0 \\
E9 & 5 & 1 & 5 & 3 & 4 & 2 & 5 & 1 & 5 & 1 & 90,0 \\
E10 & 4 & 4 & 4 & 2 & 4 & 2 & 4 & 2 & 5 & 2 & 72,5 \\
E11 & 4 & 2 & 4 & 2 & 4 & 2 & 4 & 2 & 4 & 4 & 70,0 \\
\hline
\end{tabular}

Fonte: Autores. 
Obteve-se a média de 76,59 pontos para a usabilidade que equivale a um bom resultado geral; sendo que $27 \%$ responderam “Ok”, 36\% responderam Bom e 36\% responderam Excelente, conforme Tabela 7.

Tabela 7 - Resultado da Avaliação.

\begin{tabular}{ll}
\hline Resultado SUS & Total de Avaliação \\
\hline Ok & $27 \%$ \\
Bom & $36 \%$ \\
Excelente & $36 \%$ \\
\hline
\end{tabular}

Fonte: Autores.

Os valores coletados no SUS foram normalizados, aplicando o critério apresentado na Tabela 3, descrito na metodologia, resultando a Tabela 8.

Tabela 8 - Resultado normalização.

\begin{tabular}{|c|c|c|c|c|c|c|c|c|c|c|c|c|c|c|c|c|c|c|c|c|c|c|}
\hline \multirow{3}{*}{ 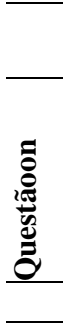 } & \multicolumn{6}{|c|}{ Product Owner } & \multicolumn{6}{|c|}{ Analista de Controle } & \multicolumn{10}{|c|}{ Operador } \\
\hline & \multicolumn{2}{|c|}{ E1 } & \multicolumn{2}{|c|}{ E2 } & \multicolumn{2}{|c|}{ E3 } & \multicolumn{2}{|c|}{ E4 } & \multicolumn{2}{|c|}{ E5 } & \multicolumn{2}{|c|}{ E6 } & \multicolumn{2}{|c|}{ E7 } & \multicolumn{2}{|c|}{ E8 } & \multicolumn{2}{|c|}{ E9 } & \multicolumn{2}{|c|}{ E10 } & \multicolumn{2}{|c|}{ E11 } \\
\hline & $\mu$ & $\lambda$ & $\mu$ & $\lambda$ & $\mu$ & $\lambda$ & $\mu$ & $\lambda$ & $\mu$ & $\lambda$ & $\mu$ & $\lambda$ & $\mu$ & $\lambda$ & $\mu$ & $\lambda$ & $\mu$ & $\lambda$ & $\mu$ & $\lambda$ & $\mu$ & $\lambda$ \\
\hline $\mathrm{Q}_{1}$ & 0,75 & 0,25 & 0,75 & 0,25 & 1,00 & 0,00 & 1,00 & 0,00 & 1,00 & 0,00 & 1,00 & 0,00 & 1,00 & 0,00 & 0,75 & 0,25 & 1,00 & 0,00 & 0,75 & 0,25 & 0,75 & 0,25 \\
\hline $\mathrm{Q}_{2}$ & 0,75 & 0,25 & 0,75 & 0,25 & 0,75 & 0,25 & 0,75 & 0,25 & 1,00 & 0,00 & 0,75 & 0,25 & 1,00 & 0,00 & 1,00 & 0,00 & 1,00 & 0,00 & 0,25 & 0,75 & |0,75 & 0,25 \\
\hline $\mathrm{Q}_{3}$ & 0,75 & 0,25 & 0,75 & 0,25 & 0,75 & 0,25 & 0,75 & 0,25 & 1,00 & 0,00 & 1,00 & 0,00 & 1,00 & 0,00 & 1,00 & 0,00 & 1,00 & 0,00 & 0,75 & 0,25 & 0,75 & 0,25 \\
\hline $\mathrm{Q}_{4}$ & 0,75 & 0,25 & 0,75 & 0,25 & 1,00 & 0,00 & 0,25 & 0,75 & 1,00 & 0,00 & 0,75 & 0,25 & 1,00 & 0,00 & 0,75 & 0,25 & 0,50 & 0,50 & 0,75 & 0,25 & | 0,75 & 0,25 \\
\hline $\mathrm{Q}_{5}$ & 0,75 & 0,25 & 0,75 & 0,25 & 0,75 & 0,25 & 0,75 & 0,25 & 1,00 & 0,00 & 0,75 & 0,25 & 0,75 & 0,25 & 0,50 & 0,50 & 0,75 & 0,25 & 0,75 & 0,25 & 0,75 & 0,25 \\
\hline $\mathrm{Q}_{6}$ & 0,25 & 0,75 & 0,25 & 0,75 & 0,25 & 0,75 & 0,00 & 1,00 & 0,00 & 1,00 & 0,75 & 0,25 & 0,75 & 0,25 & 0,75 & 0,25 & 0,75 & 0,25 & 0,75 & 0,25 & 0,75 & 0,25 \\
\hline $\mathrm{Q}_{7}$ & 0,25 & 0,75 & 0,75 & 0,25 & 0,75 & 0,25 & 0,75 & 0,25 & 1,00 & 0,00 & 0,75 & 0,25 & 1,00 & 0,00 & 0,75 & 0,25 & 1,00 & 0,00 & 0,75 & 0,25 & 0,75 & 0,25 \\
\hline $\mathrm{Q}_{8}$ & 0,75 & 0,25 & 1,00 & 0,00 & 1,00 & 0,00 & 0,75 & 0,25 & 1,00 & 0,00 & 0,75 & 0,25 & 1,00 & 0,00 & 0,75 & 0,25 & 1,00 & 0,00 & 0,75 & 0,25 & \begin{tabular}{l|l} 
& 0,77
\end{tabular} & 0,25 \\
\hline $\mathrm{Q}_{9}$ & 0,75 & 0,25 & 0,75 & 0,25 & 0,75 & 0,25 & 0,75 & 0,25 & 0,25 & 0,75 & 1,00 & 0,00 & 0,75 & 0,25 & 0,75 & 0,25 & 1,00 & 0,00 & 1,00 & 0,00 & 0,75 & 0,25 \\
\hline $\mathrm{Q}_{10}$ & 0,25 & 0,75 & 0,75 & 0,25 & 0,75 & 0,25 & 0,75 & 0,25 & 1,00 & 0,00 & 0,75 & 0,25 & 0,75 & 0,25 & 1,00 & 0,00 & 1,00 & 0,00 & 0,75 & 0,25 & 0,25 & 0,75 \\
\hline
\end{tabular}

Fonte: Autores.

Entende-se que a usabilidade está dentro de um padrão de qualidade aceitável, pois observa-se que a satisfação dos usuários, Q1, apresenta o resultado totalmente verdadeiro, assim como, o resultado da complexidade, Q2, apresenta resultado na região verdadeira $(0,75 ; 0,25)$, de acordo com a Tabela 9. 
Tabela 9 - Resultado da análise do questionário.

\begin{tabular}{c|c|c|l}
\hline \multirow{2}{*}{ Fator } & \multicolumn{2}{|c|}{$\begin{array}{c}\text { Grau } \\
\text { resultante }\end{array}$} & Decisão \\
\cline { 2 - 4 } & $\boldsymbol{\mu}$ & $\boldsymbol{\lambda}$ & \\
\hline Q1 & 1,00 & 0,00 & Aceitável \\
Q2 & 0,75 & 0,25 & Aceitável \\
Q3 & 0,75 & 0,25 & Aceitável \\
Q4 & 0,75 & 0,25 & Aceitável \\
Q5 & 0,75 & 0,25 & Aceitável \\
Q6 & 0,25 & 0,75 & Não Aceitável \\
Q7 & 0,75 & 0,25 & Aceitável \\
Q8 & 1,00 & 0,00 & Aceitável \\
Q9 & 0,75 & 0,25 & Aceitável \\
Q10 & 0,75 & 0,25 & Aceitável \\
\hline Análise Global & $\mathbf{0 , 7 5}$ & $\mathbf{0 , 2 5}$ & Aceitável \\
\hline
\end{tabular}

Fonte: Autores.

A resposta sobre Facilidade de uso, Q3, apresenta resultado na região verdadeira $(0,75 ; 0,25)$ significando que o usuário tem uma facilidade de uso, corroborando com a Q4, que significa que o usuário não apresenta dificuldade na utilização do SGPS, portanto não necessita de suporte técnico frequentemente.

As questões Q5, Q7 e Q9 apresentam resultados na região verdadeira $(0,75 ; 0,25)$ nos itens de integração, facilidade de aprendizado e confiança de uso respectivamente.

As questões Q8 (Complexidade de uso) e Q10 (Treinamento intenso), apresentam valores acima da média, sinalizando que o usuário respondeu corretamente o instrumento, pois ele não contradisse as respostas anteriores. Logo o resultado encontrase na região verdadeira $(0,75 ; 0,25)$ do QUPC, indicando satisfação dos usuários nestes itens. 
Figura 3 - Análise global do SUS.

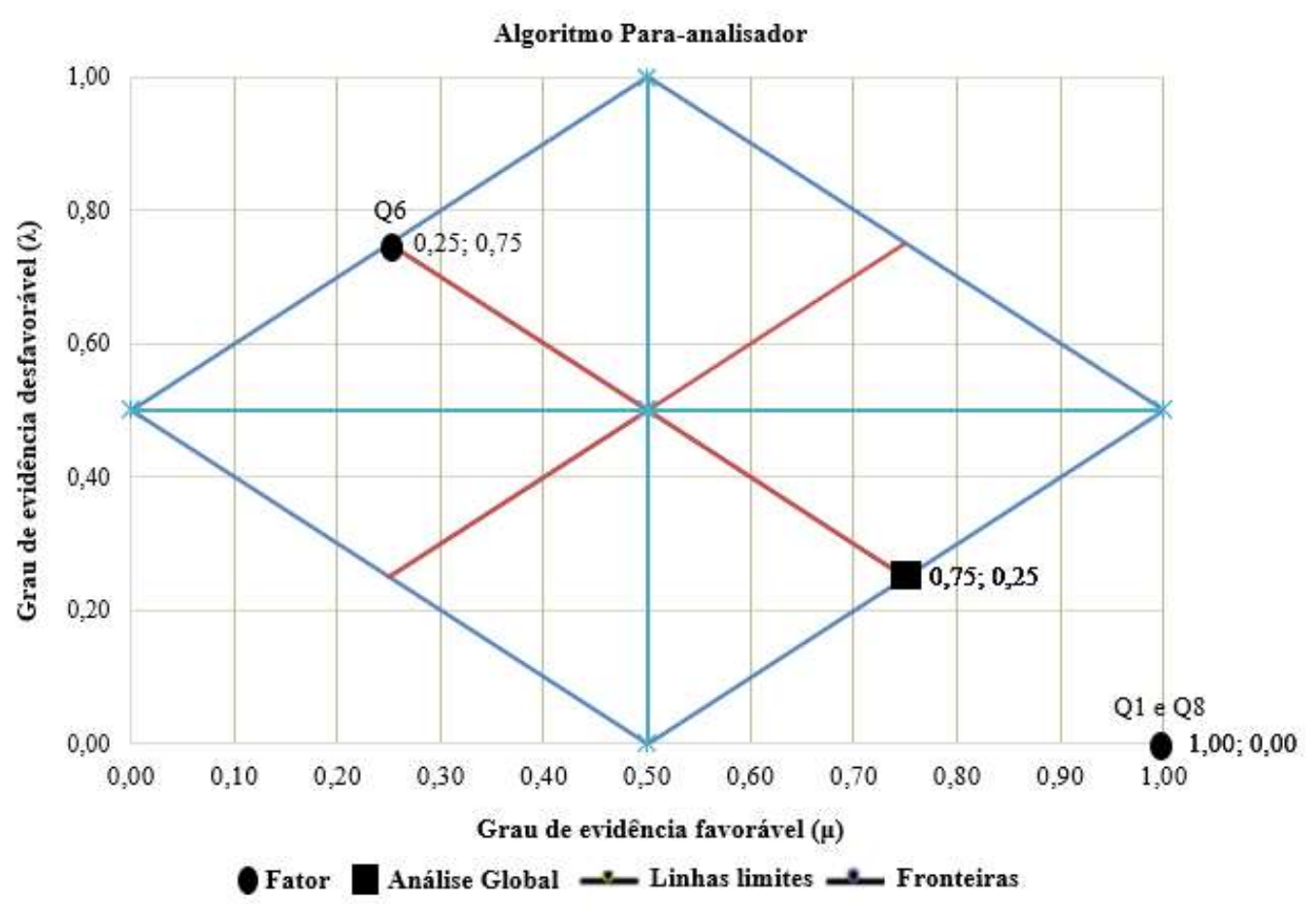

Fonte: Autores.

Os resultados de $(\mu ; \lambda)$ demonstram que os onze avaliadores apresentaram concordância em 9 fatores, sendo que dois estão na região totalmente verdadeira, ou seja, estado extremo do verdadeiro $(1,00 ; 0,00)$. Sete fatores encontram-se na região limítrofe do verdadeiro, $(0,75 ; 0,25)$, portanto as respostas para os itens Q1 a Q5 e Q7 a Q10 são representados por um único ponto, na Figura 2, devido a repetição de valores. Os resultados, do SUS e do Para-analisador, são convergentes, apresentando resultados similares. A Análise Global, resultante do Para-analisador, apresenta o par $(\mu ; \lambda)$ igual a $(0,75 ; 0,25)$, que representa um consenso na opinião dos usuários, representado no gráfico por um quadrado preto preenchido, que se encontra na região limítrofe do estado verdadeiro do QUPC.

Foi detectada uma contradição na resposta da questão Q6, apresentando um resultado de $(0,25 ; 0,75)$, localizado na região limítrofe Falsa no QUPC. Esta questão contradiz a resposta Q5, pois cinco respondentes avaliaram as duas questões com valores máximos, que pode indicar uma ausência de percepção na semântica das questões. Desta forma, pode ser constatado, na Figura 2, a contradição das respostas ao utilizar a Lógica Et e os seus operadores, que, diferentemente da análise aritmética do SUS, avalia uma única dimensão, não destacando a contradição apresentada.

A principal contribuição desta pesquisa foi a utilização de mais de uma dimensão para avaliar a usabilidade em conjunto com a Lógica $E \tau$, auxiliando em uma análise mais abrangente da usabilidade, em conjunto com valores extremos e não-extremos propostos (Abe et al., 2015).

\section{Conclusão}

Foi realizada uma adaptação do instrumento de avaliação da usabilidade de software, alicerçada pela Lógica E $\tau$, com o $\operatorname{par}(\mu, \lambda)$, sendo que as respostas pares foram atribuídas para $\lambda$, e as respostas ímpares foram atribuídas para $\mu$. Estas atribuições foram realizadas pelo fato das questões do SUS contradizerem umas as outras.

O processo de avaliação do SGPS obteve uma média de avaliação dos usuários, considerando a usabilidade boa. Não foi obtida uma pontuação mais alta, provavelmente, por falta de discernimento semântico das questões, ao utilizar o instrumento 
de avaliação. Pressupõe-se que seja necessário um treinamento do SUS, antes da sua aplicação, com os usuários da área de Gerência de Prestadores de Serviço.

A avaliação crítica dos usuários, que utilizam o SGPS, propiciou o aprimoramento da análise da usabilidade, com a Lógica $E \tau$, que apresentou a concordância em nove características, e a divergência em uma característica. Portanto, é possível utilizar a Lógica $E \tau$ em instrumentos de avaliação, a fim de explorar contradições na análise realizada com o público-alvo.

Recomenda-se que trabalhos futuros explorem outros instrumentos de avaliação, apoiados pela Lógica E $\tau$.

\section{Referências}

Abe, J. M. (7 de 2010). Paraconsistent logics and applications. 4th International Workshop on Soft Computing Applications, (pp. 11-18). 10.1109/SOFA.2010.5565631

Abe, J. M. (2015). Paraconsistent Intelligent-Based Systems - New Trends in the Applications of Paraconsistency (Vol. 94). (Springer, Ed.) Switzerland: Springer. https://doi-org.ez338.periodicos.capes.gov.br/10.1007/978-3-319-19722-7

Abe, J. M., Akama, S., \& Nakamatsu, K. (2015). Introduction to Annotated Logics: Foundations for Paracomplete and Paraconsistent Reasoning. Cham: Springer International Publishing. 10.1007/978-3-319-17912-4_1

Abe, J. M., Da Silva Filho, J. I., Celestino, U., \& Corrêa, d. A. (2011). Lógica Paraconsistente Anotada Evidencial Eq. Comunicar.

Akama, S. (2016). Towards Paraconsistent Engineering. Springer International Publishing. 10.1007/978-3-319-40418-9

Brooke, J. (11 de 1995). SUS: A quick and dirty usability scale. Usability Eval. Ind., 189.

Buyya, R., Broberg, J., \& Goscinski, A. (2011). Cloud Computing: principles and paradigms. John Wiley \& Sons.

Carvalho, F. R., \& Abe, J. M. (2018). A Paraconsistent Decision-Making Method (Vol. 87). Springer International Publishing. https://doi.org/10.1007/978-3319-74110-9

Chen, C., Lin, S., Shoga, M., Wang, Q., \& Boehm, B. (2018). How Do Defects Hurt Qualities? An Empirical Study on Characterizing a Software Maintainability Ontology in Open Source Software. (pp. 226-237). IEEE. 10.1109/QRS.2018.00036

Da Silva Filho, J. I., Abe, J. M., \& Torres, G. L. (2008). Inteligência artificial com as redes de análises paraconsistentes. LTC-Livros Técnicos e Científicos Editora SA, 313.

Denning, P. J. (8 de 2016). Software quality. Communications of the ACM, 59, 23-25. 10.1145/2971327

Esaki, K., Azuma, M., \& Komiyama, T. (2013). Introduction of Quality Requirement and Evaluation Based on ISO/IEC SQuaRE Series of Standard. Em Trustworthy Computing and Services (pp. 94-101). Springer Berlin Heidelberg. 10.1007/978-3-642-35795-4_12

Ghanbari, H., Vartiainen, T., \& Siponen, M. (2 de 2018). Omission of Quality Software Development Practices: A Systematic Literature Review. ACM Comput. Surv., 51. 10.1145/3177746

Harrison, R., Flood, D., \& Duce, D. (2013). Usability of mobile applications: literature review and rationale for a new usability model. Journal of Interaction Science, 1, 1. 10.1186/2194-0827-1-1

ISO/IEC 25010. (15 de 1 de 2021). Systems and software engineering — Systems and software Quality Requirements and Evaluation (SQuaRE) — System and software quality models. Systems and software engineering - Systems and software Quality Requirements and Evaluation (SQuaRE) - System and software quality models. Fonte: https://www.iso.org/standard/35733.html.

ISO/IEC 25040. (15 de 1 de 2021). Systems and software engineering — Systems and software Quality Requirements and Evaluation (SQuaRE) — Evaluation process. Systems and software engineering - Systems and software Quality Requirements and Evaluation (SQuaRE) - Evaluation process. Fonte: https://www.iso.org/standard/35765.html.

Jeff Sauro, J. R. (2016). Quantifying the User Experience. Elsevier.

Kniberg, H. (11 de 2015). Scrum and XP from the Trenches (Enterprise Software Development). lulu.com; Annotated edition.

Laudon, J. P. (2014). Management information systems: Managing the Digital Firm (13th ed.). Pearson.

Manchar, A., \& Chouhan, A. (2 de 2017). Salesforce CRM: A new way of managing customer relationship in cloud environment., (pp. 1-4). 10.1109/ICECCT.2017.8117887

Nakai, H., Tsuda, N., Honda, K., Washizaki, H., \& Fukazawa, Y. (2016). Initial Framework for Software Quality Evaluation Based on ISO/IEC 25022 and ISO/IEC 25023. (pp. 410-411). IEEE. 10.1109/QRS-C.2016.66

Pereira, A. S., Shitsuka, D. M., Parreira, F. J., \& Shitsuka, R. (2018). Metodologia da pesquisa científica. Fonte: http://repositorio.ufsm.br/handle/1/15824

Shen, P., Ding, X., Ren, W., \& Yang, C. (2018). Research on Software Quality Assurance Based on Software Quality Standards and Technology Management. (pp. 385-390). IEEE. 10.1109/SNPD.2018.8441142

Wahyuningrum, T., Kartiko, C., \& Wardhana, A. C. (10 de 2020). Exploring e-Commerce Usability by Heuristic Evaluation as a Compelement of System Usability Scale. 2020 International Conference on Advancement in Data Science, E-learning and Information Systems (ICADEIS). IEEE. 10.1109/icadeis49811.2020.9277343 